\title{
Foreword
}

\section{Giancarlo Ruocco: from inelastic X-ray scattering to neuroscience}

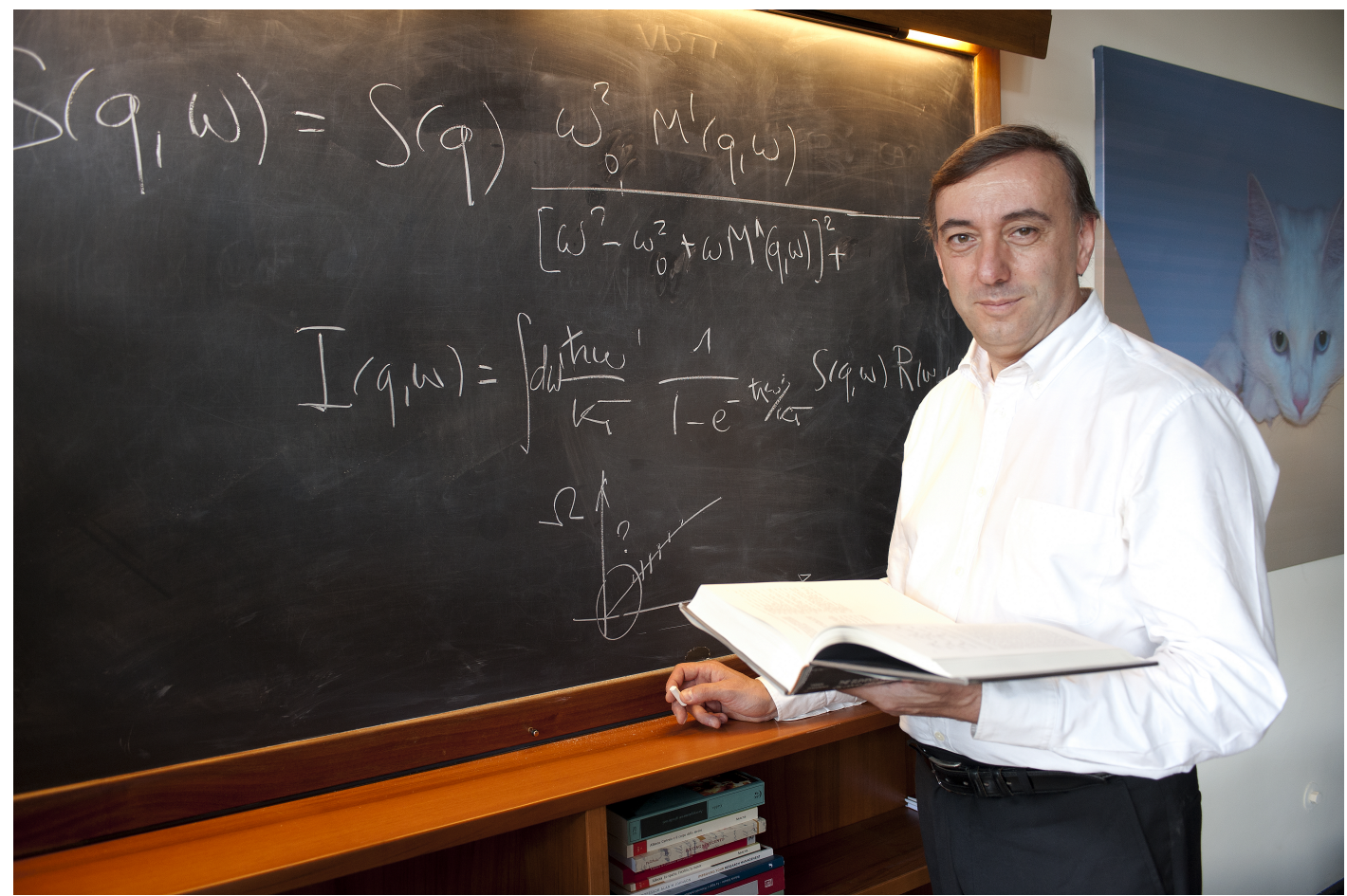

This Special issue of the "Condensed Matter Physics" journal is dedicated to Professor Giancarlo Ruocco on the occasion of his 60 birthday. Giancarlo Ruocco is widely known as one of the pioneers in the development of the meV resolution Inelastic X-ray Scattering (IXS), a novel and yet state-ofthe-art technique for studying atomistic dynamics in condensed and soft matter. The IXS opened new possibilities in understanding the microscopic nature of relaxation processes in liquids, glasses, complex biomolecular systems and polymers. His strong background in theory allowed Giancarlo Ruocco to combine experimental findings with theoretical analysis of IXS data, that led to fundamental results for dynamic processes in various condensed and soft matter systems.

Born in Rome, Giancarlo graduated in 1981 with Laurea degree in Physics from the Sapienza University of Rome. His first studies were performed under the guidance of Prof. G. Signorelli and Prof. V. Mazzacurati and were focused on Raman scattering in water. In 1984 Giancarlo took a position of Assistant Professor in Condensed Matter Physics at Department of Physics, L'Aquila University, followed by the position of Associate Professor (1992-2000) at the same University. Since 2000 Giancarlo Ruocco was holding the Full Professor position in Condensed Matter Physics at his Alma Mater, the Sapienza University of Rome. In 2000-2004 he was a leader of the "GLAS" (Liquids and Amorphous Solid) group at the Department of Physics, which was transformed into the newly created Research Center "SOFT" of the INFM with Giancarlo being the director. In November 2007 Giancarlo Ruocco was elected as the director of the Physics Department at Sapienza University of Rome. In the period 2010-2014 he was Vice-Rector of the Sapienza University responsible for the Research Policy.

The new direction in the Giancarlo's scientific career and his new challenge was connected with an interdisciplinary Center for Life Nano Science (CLNS)@Sapienza, a part of the Italian Institute 
of Technology, with the main mission of CLNS being the study of neurodegenerative diseases with a multifaceted approach: from nanotechnology to biochemistry, from optics to molecular biology. The importance of the research at CLNS was supported by funding the first five (2011-2015) years (20 MEuro) and recently re-funded for five more years (2016-2020). To the date Giancarlo Ruocco is the coordinator of CLNS.

Over the years the scientific interests of Giancarlo permanently broadened: starting from Raman scattering and IXS, dynamics of liquids, glasses and soft matter to the neurosciences and neurodegenerative disease. Now he is the responsible person for the technical development of a long working distance microscope for the detection in the human retina of those small aggregates of protein (beta-Amyloid, Tau, alpha-synuclein, ... ) which are the landmark of the neurodegenerative diseases, aiming at their early detection. He is also responsible for the development of newly conceived frequency filters for high throughput Brillouin microscopy, a technique that has been already applied to study the biomechanics of stress-granules in iPSC motoneurons from ALS patients.

We would like to recall here several most important developments and scientific results by Giancarlo.

In 1998 in collaboration with Francesco Sette (ESRF, Grenoble) Giancarlo published in Science magazine a paper on the new IXS technique [1]. Specifically its application was to crack a fundamental issue concerning the physics of glasses, which was an open problem in the late 1990s. While the existence of hypersonic waves was somehow rationalized in liquids, the possible existence of equivalent excitations in glasses was highly debated. Hence, the first observation of propagating acoustic phonon-like excitations in glasses and glass-forming liquids down to wavelengths comparable with the interparticle distance [2] was a real breakthrough for the whole science of disordered materials.

A second groundbreaking result concerns the nature of the so-called "fast sound" in water, i.e., the propagation of nanometer-wavelength excitations with a sound velocity value doubling the hydrodynamic value [3]. Insights from Inelastic Neutron Scattering and molecular simulations ignited a long lasting debate on the origin of such a process. One proposal, based on a scenario shared with disparate mass binary mixtures, ascribed such high frequency waves to excitations propagating on the network of the light species. Another proposal advanced the idea that when the energy of the "normal sound" mode (slope of about $1.5 \mathrm{~km} / \mathrm{s}$ ) reaches an energy of $\sim 5 \mathrm{meV}$ (upon decreasing its wavelength), it would "interact" with the dispersionless transverse mode located at that energy. Basically the branch linearly dispersing at low wave numbers $q$, bends and becomes, at high $q$, almost dispersionless. The other branch, dispersionless at low $q$, acquires, at high $q$, a linear dispersion with a sound velocity larger with respect to the sound velocity of the other branch at low $q$. Critically, IXS measurements demonstrated that the doubling of the sound velocity is actually due to a viscoelastic transition from a low frequency, liquid-like regime, to a solid-like high frequency sound, corresponding indeed to the sound velocity of ice, the solid form of water [4]. Taken together these two contributions established the equivalence of liquids and glasses as far as the behaviour of collective acoustic modes is concerned.

Later in 2003, the idea of a connection between the properties of liquids and glasses was supported by experimental evidences published in a new Science paper, which established a correspondence between the viscosity of a glass-forming liquid nearby the glass transition temperature $\left(T_{\mathrm{g}}\right)$ and the vibrational properties of the corresponding glass well below $T_{\mathrm{g}}$ [5]. Specifically, a correlation was found between the fragility, the steepness index of the logarithmic viscosity in an inverse temperature scale, and the nonergodicity factor of the glass in the low-temperature, harmonic, regime. The important straightforward consequence, i.e., the extension of the fragility concept to the glassy state as this work, indicated how to determine the fragility uniquely from glass properties well below $T_{\mathrm{g}}$.

Moving from high frequency dynamics to slow structural rearrangements in supercooled liquids, Giancarlo suggested a topological interpretation for the critical slowing down of structural dynamics. In a series of works with Francesco Sciortino, the concept of inherent saddles was introduced. This provided a new insight on the meaning of the critical divergence of structural relaxation time by connecting it to the number of negative eigenvalues associated with the closest stationary points in the potential energy landscape [6]. Expanding his interests from equilibrium slow dynamics to non-equilibrium aging dynamics in glass forming systems and soft matter, Giancarlo also gave important contributions to the understanding of effective temperatures in off-equilibrium systems. Within a collaboration with Giorgio Parisi, he studied the aging behaviour of an original glass former model undergoing a rapid density crunch across the glass transition temperature. Giancarlo and collaborators demonstrated that the following off- 
equilibrium dynamics obeyed a generalized form of the fluctuation dissipation theorem characterized by an effective temperature that can be anticipated from an equilibrium determination of the glass transition line [7]. Moving from hard to soft matter, Giancarlo supervised and coordinated a wide spectrum of experimental work addressing the nature of dynamical arrest and rheological properties of colloidal gels.

Collective dynamics of glasses in low-frequency region was in focus of many simulation and theoretical studies by Giancarlo and his collaborators. We would like to mention here a brilliant suggestion made by Giancarlo on macroscopic speed of sound in simple one-component glasses. In [8], the macroscopic speed of sound was defined as $c_{0}=\left[k_{\mathrm{B}} T / m S^{\prime}(q \rightarrow 0)\right]^{1 / 2}$ with $S^{\prime}(q)=S(q)-f(q)$, where $S(q)$ is the regular static structure factor, $f(q)$ is the non-ergodicity factor, and $k_{\mathrm{B}}, T$ and $m$ are Boltzmann constant, temperature and atomic mass, respectively. Remarkably, the similar expression for the speed of sound in simple glasses was proposed by Götze and Mayr independently the same year [9].

A large part of important theoretical results, based on heterogeneous-elasticity theory [10], was obtained on the anomalous vibrational properties of glasses. The first result was a relation of the excess of the vibrational density of states over Debye's $\omega^{2}$ law ("boson peak") with the sound attenuation of glasses [11]. Intensifying the discussions on the vibrational properties of glasses, the approach was generalized for correlated elasticity fluctuations [12], inclusion of anharmonicity [13], and strong fluctuations [14]. In particular, the interplay between marginal stability and anharmonicity was shown to give rise to a fractal frequency dependence of the sound attenuation below the boson peak, which was observed experimentally in glassy $\mathrm{GeO}_{2}$ [15] and in numerical simulations of glasses [16, 17]. Another interest of Giancarlo in this context was to discuss and solve a simplified mass-spring model, which was named "Euclidean random matrix" model [18, 19]. For this model, a moment-conserving mean-field theory was derived [20] and a cross-link to heterogeneous-elasticity theory was established [21].

Supercritical fluids have been considered for a long time as systems with smoothly changing properties by density/pressure increase from rarefied to very dense states. In [22], Giancarlo with coauthors pointed out that for supercritical oxygen from IXS experiment there was observed a typical for dense liquids "positive sound dispersion" (PSD), which is a high-frequency deviation of the dispersion and an apparent propagation speed of acoustic modes from the hydrodynamic linear dispersion and macroscopic adiabatic speed of sound outside the hydrodynamic region. This was the first paper in which the idea was proposed to discriminate between gas-like and liquid-like supercritical fluids on the basis of collective dynamics. Later on, the IXS experiments on supercritical Ar and molecular dynamics simulations [23] allowed one to estimate the decrease of the PSD with the pressure drop practically to the vanishing PSD and to suggest that the PSD can be the quantity discriminating between the liquid- and gas-like supercritical fluids. An obvious suggestion was made in [23] that the gas-liquid coexistence curve below the critical point could be extended into the separation line between the gas-like and liquid-like supercritical fluids, which is the Widom line emanating from the critical point. In [24], it was clarified that the separation line between the gas-like and liquid-like supercritical fluids can be extended to very high temperatures following the line of minima of thermal diffusivity and kinematic viscosity, which by approaching the critical point transforms into the Widom line, which was also supported by the theory of positive sound dispersion in supercritical fluids [25].

Another part of Giancarlo's numerous topics of interest is the propagation and localization of light in random media [26, 27]. He established a special lab at CLNS for investigating fibres with transverse disorder, which were found to exhibit transverse localization. The samples were found to exhibit channels of single-mode character [28] with the radius of the transverse localization length. Looking for an analytical description of these phenomena [29], it was found that the basic equations used so far in the literature for describing light in a disordered medium predicted a strong wavelength dependence of the localization radius, which was not found experimentally. Looking at the original derivation of this equation of motion from Maxwell's equations it was found that a term was dropped in order to have a Hamiltonian which looked similar to that of an electron in a random potential. This procedure can be avoided, if the equations are derived to give equations analogous to heterogeneous elasticity theory. This derivation is exact and does not predict a wave-number dependent localization length [30].

In addition to being an exceptional and creative researcher, Giancarlo has always been a great mentor who has inspired and supported the independent growth of the numerous disciples and young researchers he has supervised. The Guest Editors and the contributors of this Special issue, the Editorial Board of "Condensed Matter Physics", his numerous colleagues and friends would like to wish Giancarlo Ruocco 
a very Happy Birthday and to acknowledge his exceptional contributions to science, although we all know that his best is yet to come.

Taras Bryk (Institute for Condensed Matter Physics of NASU, Lviv, Ukraine)

Roberto Di Leonardo (Department of Physics, Sapienza University of Rome, Rome, Italy)

Walter Schirmacher (Johannes Gutenberg University of Mainz, Mainz, Germany)

Tullio Scopigno (Department of Physics, Sapienza University of Rome, Rome, Italy)

\section{References}

1. Sette F., Krisch M.H., Masciovecchio C., Ruocco G., Monaco G., Science, 1998, 280, 1550-1555, doi $10.1126 /$ science.280.5369.1550

2. Benassi P., Krisch M., Masciovecchio C., Mazzacurati V., Monaco G., Ruocco G., Sette F., Verbeni R., Phys. Rev. Lett., 1996, 77, 3835, doi 10.1103/PhysRevLett.77.3835

3. Ruocco G., Sette F., Bergmann U., Krisch M., Masciovecchio C., Mazzacurati V., Signorelli G., Verbeni R., Nature, 1996, 379, 521, doi $10.1038 / 379521 \mathrm{a} 0$

4. Sette F., Ruocco G., Krisch M., Masciovecchio C., Verbeni R., Bergmann U., Phys. Rev. Lett., 1996, 77, 83, doi $10.1103 /$ PhysRevLett.77.83

5. Scopigno T., Ruocco G., Sette F., Monaco G., Science, 2003, 302, 849-852, doi $10.1126 /$ science.1089446

6. Angelani L., Di Leonardo R., Ruocco G., Scala A., Sciortino F., Phys. Rev. Lett., 2000, 85, 5356, doi $10.1103 /$ PhysRevLett.85.5356

7. Di Leonardo R., Angelani L., Parisi G., Ruocco G., Phys. Rev. Lett., 2000, 84, 6054, doi 10.1103/PhysRevLett.84.6054.

8. Ruocco G., Sette F., Di Leonardo R., Monaco G., Sampoli M., Scopigno T., Viliani G., Phys. Rev. Lett., 2000, 84, 5788, doi $10.1103 /$ PhysRevLett.84.5788

9. Götze W., Mayr M.R., Phys. Rev. E, 2000, 61, 587, doi 10.1103/PhysRevE.61.587.

10. Schirmacher W., Europhys. Lett., 2006, 73, 892, doi:10.1209/epl/i2005-10471-9

11. Schirmacher W., Ruocco G., Scopigno T., Phys. Rev. Lett., 2007, 98, 025501, doi $10.1103 /$ PhysRevLett.98.025501

12. Schirmacher W., Schmid B., Tomaras C., Viliani G., Baldi G., Ruocco G., Scopigno T., Phys. Status Solidi C, 2008, 5, 862, doi $10.1002 /$ pssc. 200777584

13. Marruzzo A., Köhler S., Fratalocchi A., Ruocco G., Schirmacher W., Eur. Phys. J. Spec. Top., 2013, 216, 83, doi $10.1140 /$ epjst/e2013-01731-5

14. Köhler S., Ruocco G., Schirmacher W., Phys. Rev. B, 2013, 88, 064203, doi $10.1103 /$ PhysRevB.88.064203

15. Ferrante C., Pontecorvo E., Cerullo G., Chiasera A., Ruocco G., Schirmacher W., Scopigno T., Nat. Commun., 2013, 4, 1793, doi $10.1038 /$ ncomms2826.

16. Marruzzo A., Schirmacher W., Fratalocchi A., Ruocco G., Sci. Rep., 2013, 3, 1407, doi 10.1038/srep01407

17. Mizuno H., Ruocco G., Mossa S., Preprint arXiv:1905.10235, 2019.

18. Grigera T.S., Martín-Mayor V., Parisi G., Verrocchio P., Phys. Rev. Lett., 2001, 87, 085502, doi $10.1103 /$ PhysRevLett.87.085502

19. Ganter C., Schirmacher W., Phys. Rev. B, 2010, 82, 094205, doi 10.1103/PhysRevB.82.094205

20. Folli V., Ruocco G., Schirmacher W., Front. Phys., 2017, 5, 29, doi $10.3389 /$ fphy.2017.00029.

21. Schirmacher W., Folli V., Ganter C., Ruocco G., J. Phys. A: Math. Theor., doi $10.1088 / 1751-8121 / a b 4 a 35$, (in print).

22. Gorelli F., Santoro M., Scopigno T., Krisch M., Ruocco G., Phys. Rev. Lett., 2006, 97, 245702, doi 10.1103/PhysRevLett.97.245702

23. Simeoni G.G., Bryk T., Gorelli F.A., Krisch M., Ruocco G., Santoro M., Scopigno T., Nat. Phys., 2010, 6, 503, doi $10.1038 /$ nphys 1683

24. Gorelli F.A., Bryk T., Krisch M., Ruocco G., Santoro M., Scopigno T., Sci. Rep., 2013, 3, 1203, doi $10.1038 /$ srep01203.

25. Bryk T., Mryglod I., Scopigno T., Ruocco G., Gorelli F., Santoro M., J. Chem. Phys., 2010, 133, 024502, doi $10.1063 / 1.3442412$

26. Angelani L., Conti C., Ruocco G., Zamponi F., Phys. Rev. Lett., 2006, 96, 065702, doi $10.1103 /$ PhysRevLett.96.065702

27. Conti C., Angelani L., Ruocco G., Phys. Rev. A, 2007, 75, 033812, doi $10.1103 /$ PhysRevA.75.033812 
28. Ruocco G., Abaie B., Schirmacher W., Mafi A., Leonetti M., Nat. Commun., 2017, 8, 14571, doi $10.1038 /$ ncomms 14571

29. Schirmacher W., Leonetti M., Ruocco G., J. Opt., 2017, 19, 045602, doi 10.1088/2040-8986/aa61c0

30. Schirmacher W., Abaie B., Mafi A., Ruocco G., Leonetti M., Phys. Rev. Lett., 2018, 120, 067401, doi $10.1103 /$ PhysRevLett.120.067401 